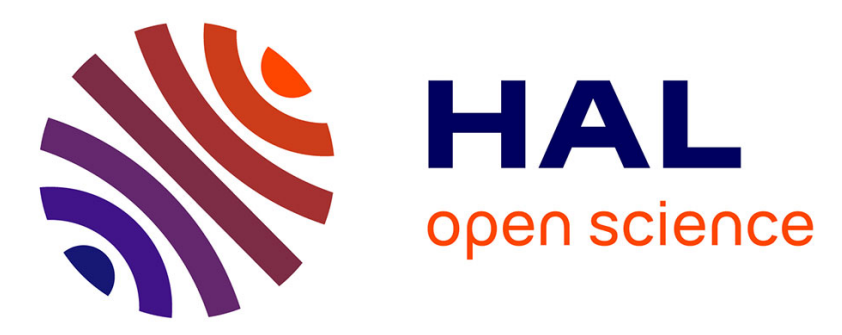

\title{
Détermination de la répartition de potentiel dans l'espace interélectrode d'une sonde à grille plongée dans un plasma maxwellien
}

\author{
J.-C. Braun, Murielle Richard, M. Felden
}

\section{- To cite this version:}

J.-C. Braun, Murielle Richard, M. Felden. Détermination de la répartition de potentiel dans l'espace interélectrode d'une sonde à grille plongée dans un plasma maxwellien. Journal de Physique, 1973, 34 (10), pp.859-867. 10.1051/jphys:019730034010085900 . jpa-00207448

\section{HAL Id: jpa-00207448 \\ https://hal.science/jpa-00207448}

Submitted on 1 Jan 1973

HAL is a multi-disciplinary open access archive for the deposit and dissemination of scientific research documents, whether they are published or not. The documents may come from teaching and research institutions in France or abroad, or from public or private research centers.
L'archive ouverte pluridisciplinaire HAL, est destinée au dépôt et à la diffusion de documents scientifiques de niveau recherche, publiés ou non, émanant des établissements d'enseignement et de recherche français ou étrangers, des laboratoires publics ou privés. 


\title{
DÉTERMINATION DE LA RÉPARTITION DE POTENTIEL DANS L'ESPACE INTERÉLECTRODE D'UNE SONDE A GRILLE PLONGÉE DANS UN PLASMA MAXWELLIEN
}

\author{
J.-C. BRAUN, M. RICHARD et M. FELDEN \\ Laboratoire de Physique des Milieux Ionisés, Université Nancy I, \\ Boulevard des Aiguillettes, Case Officielle 140, 54037 Nancy, Cédex, France
}

(Reçu le 27 septembre 1972, révisé le 1er juin 1973)

\begin{abstract}
Résumé. - Nous étudions différents cas possibles de répartition de potentiel entre grille et électrode collectrice d'une sonde à grille plongée dans un plasma maxwellien. Cette détermination analytico-numérique se fait à partir de l'équation de Poisson, le modèle choisi étant unidimensionnel. Les résultats exposés sont ceux obtenus dans le cas où le potentiel est monotone, puis dans celui où il présente un extrémum dans l'espace interélectrode.
\end{abstract}

\begin{abstract}
We study different possible forms of the potential in the space between the grid and the collecting electrode of a grid probe immersed in a maxwellian plasma. The spatial variation of the potential is determined by a numerical-analysis solution of the Poisson equation in one dimension. The results are those obtained in the cases of a monotonic potential and of a potential with an extremum in the interelectrode space.
\end{abstract}

Considérons un plasma maxwellien isotrope, sans collision, en équilibre thermodynamique, dans lequel est plongée une sonde à grille constituée de deux électrodes planes (Fig. 1) : la grille et le collecteur, portées respectivement aux potentiels $V_{\mathrm{g}}$ et $V_{\mathrm{c}}$ par rapport au potentiel de plasma. Il est alors intéressant de connaître la répartition de potentiel dans l'espace interélectrode puisqu'elle permet de déterminer les densités de courant électronique et ionique dans ce même espace ; cette détermination faite sur le collecteur entraîne la connaissance des courants collectés par la sonde en fonction des paramètres $V_{\mathrm{c}}$ et $V_{\mathrm{g}}$. Nous verrons en effet que suivant le type de répartition de potentiel (monotone, avec maximum ou avec minimum), les densités de courant au niveau de la sonde n'ont pas les mêmes formes analytiques. Le problème est le suivant : quel est le type de répartition de potentiel qui correspond à deux valeurs $V_{\mathrm{g}}$ et $V_{\mathrm{c}}$ des potentiels de grille et de collecteur ?

L'étude théorique que nous exposons permet de dresser une carte dans le plan $\left(V_{\mathrm{g}}, V_{\mathrm{c}}\right)$, donnant à l'expérimentateur les domaines où il peut espérer une véritable séparation des charges avec la sonde à grille utilisée.

La théorie complète est rendue difficile par l'existence d'une gaine au voisinage de la grille. L'épaisseur de cette région de transition est habituellement de l'ordre de la longueur de Debye et le potentiel y varie très rapidement depuis le potentiel de plasma

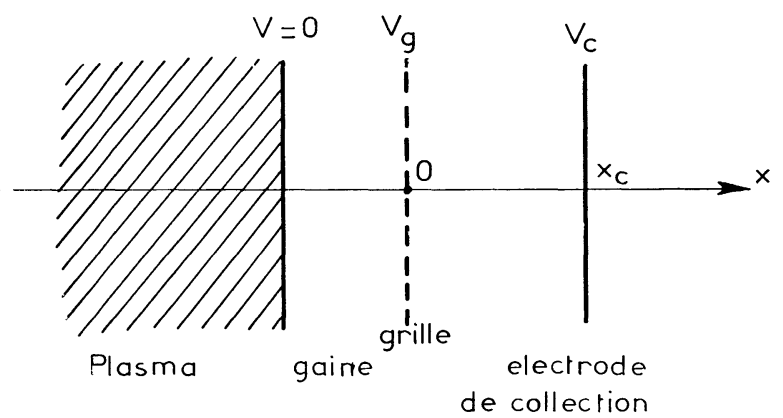

Fig. 1. - Représentation schématique de la sonde à grille plongée dans le plasma.

jusqu'au potentiel de grille. Ces deux remarques justifient l'approximation que nous avons faite et qui consiste à remplacer le phénomène de gaine par une discontinuité de potentiel au niveau de la grille.

Une deuxième approximation, justifiée par l'existence d'une direction privilégiée (celle du champ électrique, normale aux plans des électrodes, entre grille et collecteur) nous permet de considérer que le problème est unidimensionnel.

La méthode de résolution utilisée consiste à obtenir une intégrale première de l'équation de Poisson, puis d'intégrer numériquement l'équation ainsi obtenue. Des travaux semblables [1], [2] ont conduit les auteurs à la détermination du potentiel entre les deux électrodes d'une diode, mais une méthode numérique 
élémentaire leur permettait seulement de calculer une distance interélectrode après s'être donné la constante d'intégration représentée par le carré du champ électrostatique sur la grille. La méthode que nous avons utilisée est une "méthode du tir » permettant de déterminer cette constante d'intégration après s'être imposé la distance interélectrode.

Enfin, nous avons fait les hypothèses simplificatrices suivantes :

- Les températures électronique et ionique sont égales et le plasma devant la grille est supposé neutre (les densités électronique et ionique sont alors égales dans le plasma).

1. Mise en équation du problème. - Le potentiel $V(x)$ à la distance $x$ de la grille est solution de l'équation de Poisson :

$$
\frac{\mathrm{d}^{2} V(x)}{\mathrm{d} x^{2}}=\frac{e}{\varepsilon_{0}}\left[N_{\mathrm{e}}(x)-N_{\mathrm{i}}(x)\right]
$$

$V(x)$ est mesuré par rapport au potentiel du plasma, $N_{\mathrm{e}}(x)$ et $N_{\mathrm{i}}(x)$ sont les densités électronique et ionique à la distance $x$, calculées à partir des fonctions de distribution correspondantes :

$$
N_{\mathrm{j}}(x)=N_{0} \int f_{\mathrm{j}}(x, v) \mathrm{d} v \quad \text { où } \quad j=e \quad \text { ou } \quad i
$$

$N_{0}$ étant la densité électronique et ionique dans le plasma supposé neutre devant la grille.

Les fonctions de distribution, normées à l'unité dans le plasma, sont solutions des équations de Vlasov qui, en l'absence de collisions, s'écrivent pour chaque espèce $j=e$ ou $i\left(\varepsilon_{\mathrm{e}}=-1, \varepsilon_{\mathrm{i}}=+1\right)$

$$
v \frac{\partial f_{\mathrm{j}}}{\partial x}+\varepsilon_{\mathrm{j}} \frac{e}{m_{\mathrm{j}}} \frac{\mathrm{d} V}{\mathrm{~d} x} \cdot \frac{\partial f_{\mathrm{j}}}{\partial v}=0
$$

Ces équations imposent simplement à $f_{\mathrm{j}}$ d'être fonction de l'énergie totale $\frac{1}{2} m_{\mathrm{j}} v^{2}+\varepsilon_{\mathrm{j}} e V(x)$.

Le plasma, dans lequel est plongée la sonde, étant supposé maxwellien dans l'espace interélectrode, les fonctions de distribution sont de la forme :

$$
f_{\mathrm{j}}(x, v)=\left(\frac{m_{\mathrm{j}}}{2 \pi k T_{\mathrm{j}}}\right)^{1 / 2} \exp \left[-\frac{m_{\mathrm{j}} v^{2}}{2 k T_{\mathrm{j}}}-\varepsilon_{\mathrm{j}} \frac{\mathrm{e} V}{k T_{\mathrm{j}}}\right] .
$$

2. Méthode de résolution. - Les densités sont obtenues à partir des intégrales (2) dans lesquelles les bornes, pour la vitesse $v$, dépendent des conditions de potentiel.

En utilisant les variables réduites suivantes :

$$
\begin{aligned}
\xi & =\frac{x}{\lambda_{\mathrm{D}}} & \eta(\xi) & =\frac{e V(x)}{k T} \\
n_{\mathrm{e}}(\xi) & =\frac{N_{\mathrm{e}}(x)}{N_{0}} & n_{\mathrm{i}}(\xi) & =\frac{N_{\mathrm{i}}(x)}{N_{0}}
\end{aligned}
$$

( $T$ est la température commune aux ions et aux électrons et $\lambda_{\mathrm{D}}$ la longueur de Debye) et en introduisant, comme l'ont fait Auer [1] et Mac Intyre [2], [3] le carré du champ réduit :

$$
F(\eta)=\left[\eta^{\prime}(\xi)\right]^{2}
$$

l'équation de Poisson s'écrit :

$$
F^{\prime}(\eta)=2\left[n_{\mathrm{e}}(\xi)-n_{\mathrm{i}}(\xi)\right] .
$$

La relation de définition (6) permet alors de relier $\xi$ et $\eta$ par une intégrale :

$$
\xi=-\int_{\eta_{\mathbf{g}}}^{\eta}\left[F\left(\eta^{\prime}\right)\right]^{-1 / 2} \mathrm{~d} \eta^{\prime} .
$$

Cette intégration, plus ou moins délicate suivant la complexité de la répartition de potentiel, se fait numériquement à partir de la fonction $F(\eta)$ obtenue en intégrant analytiquement l'éq. (7). Elle fait intervenir une constante que l'on doit déterminer par la condition :

$$
\xi_{\mathrm{c}}=-\int_{\eta_{\mathrm{g}}}^{\eta_{\mathrm{c}}}[F(\eta)]^{-1 / 2} \mathrm{~d} \eta
$$

la distance interélectrode réduite $\xi_{\mathrm{c}}$ étant imposée.

Cette détermination se fait en appliquant une méthode connue en analyse numérique sous le nom de «méthode de tir» [4].

3. Résultats analytiques. - La forme analytique trouvée pour la fonction $F(\eta)$ diffère suivant les valeurs de $\eta_{\mathrm{g}}$ et $\eta_{\mathrm{c}}$. Les raisonnements étant semblables dans les différents cas étudiés, nous allons donner, à titre d'exemple, la détermination de la fonction $F(\eta)$ dans le cas d'une répartition monotone de potentiel pour laquelle $V_{\mathrm{c}}<0<V_{\mathrm{g}}$; puis nous nous contenterons de donner ensuite un tableau résumant tous les résultats obtenus pour les trois grands cas de répartition suivants: potentiel monotone, potentiel avec maximum, potentiel avec minimum.

Dans le cas $\eta_{\mathrm{c}}<0<\eta_{\mathrm{g}}$ que nous avons choisi de développer ici, il est nécessaire de distinguer deux régions dans l'espace interélectrode :

Région 1, $0<\eta<\eta_{\mathrm{g}}$. - Les électrons issus du plasma arrivant sur la grille portée au potentiel positif $V_{\mathrm{g}}$ acquièrent l'énergie cinétique correspondante qu'ils vont perdre progressivement au bénéfice de leur énergie potentielle, leur vitesse est donc comprise entre $\sqrt{2 e v / m_{\mathrm{e}}}$ et l'infini ; par ailleurs, coexistent dans cette région ceux des électrons qui ont été réfléchis dans la région 2 et qui ont alors des vitesses comprises entre

$$
-\sqrt{2 e\left(V-V_{\mathrm{c}}\right) / m_{\mathrm{e}}} \text { et }-\sqrt{2 e V / m_{\mathrm{e}}} ;
$$

on obtient la densité électronique réduite suivante :

$$
n_{e_{1}}(\eta)=\frac{1}{2} \cdot e^{\eta}\left[1-2 \operatorname{erf}(\sqrt{\eta})+\operatorname{erf}\left(\sqrt{\eta-\eta_{c}}\right)\right] .
$$


Région 2, $\eta_{\mathrm{c}}<\eta<0$. - Les électrons provenant directement du plasma sont freinés dans cette région; au point $x$ où le potentiel est $V(x)$, seuls les électrons possédant une énergie cinétique supérieure à $-e V$ poursuivent leur trajectoire, les autres sont repoussés et constituent le flux des électrons réfléchis ; les vitesses vont donc varier de $-\sqrt{2 e\left(V-V_{\mathrm{c}}\right) / m_{\mathrm{e}}}$ à l'infini et la densité électronique réduite devient dans ce cas :

$$
n_{\mathrm{e} 2}(\eta)=\frac{1}{2} e^{\eta}\left[1+\operatorname{erf}\left(\sqrt{\eta-\eta_{\mathrm{c}}}\right)\right] .
$$

Pour les ions, les effets sont identiques dans les régions 1 et 2 ; seuls les ions issus du plasma et ayant une énergie cinétique supérieure à $e V_{\mathrm{g}}$ franchissent la grille, ils acquièrent alors l'énergie potentielle correspondante, au détriment de leur énergie cinétique ; ils sont ensuite accélérés en perdant progressivement cette énergie potentielle ; ainsi, au point d'abscisse $x$, leur vitesse varie de $\sqrt{2 e\left(V_{\mathrm{g}}-V\right) / m_{\mathrm{i}}}$ à l'infini et la densité ionique prend la forme suivante :

$$
n_{\mathrm{i}}(\eta)=\frac{1}{2} e^{-\eta}\left[1-\operatorname{erf}\left(\sqrt{\eta_{\mathrm{g}}-\eta}\right)\right] .
$$

En tenant compte des relations (10), (11) et (12), nous intégrons alors l'éq. (7) au moyen des intégrales suivantes :

$$
\begin{aligned}
& \int e^{\eta} \operatorname{erf}(\sqrt{\eta-a}) \mathrm{d} \eta=\mathrm{e}^{\eta} \operatorname{erf}(\sqrt{\eta-a})- \\
&-2 e^{a} \sqrt{\frac{\eta-a}{\pi}} \\
& \int \mathrm{e}^{-\eta} \operatorname{erf}(\sqrt{b-\eta}) \mathrm{d} \eta=\mathrm{e}^{-\eta} \operatorname{erf}(\sqrt{b-\eta})- \\
&-2 e^{-b} \sqrt{\frac{b-\eta}{\pi}} .
\end{aligned}
$$

En réalisant la continuité du champ électrique au point $\eta=0$, les carrés du champ réduit s'écrivent dans les régions 1 et 2

$$
\begin{aligned}
& F_{1}(\eta)=H(\eta)-2 e^{\eta} \operatorname{erf}(\sqrt{\eta})+4 \sqrt{\frac{\eta}{\pi}}+F\left(\eta_{\mathrm{g}}\right) \\
& F_{2}(\eta)=H(\eta)+F\left(\eta_{\mathrm{g}}\right)
\end{aligned}
$$

où $F\left(\eta_{\mathrm{g}}\right)$ est une constante d'intégration représentant le carré du champ réduit sur la grille et où $H(\eta)$ est une fonction commune de la forme :

$$
\begin{aligned}
H(\eta)=\exp (\eta) & {\left[1+\operatorname{erf}\left(\sqrt{\eta-\eta_{\mathrm{c}}}\right)\right]+\exp (-\eta)\left[1-\operatorname{erf}\left(\sqrt{\eta_{\mathrm{g}}-\eta}\right)\right]+\frac{2}{\sqrt{\pi}} \exp \left(\eta_{\mathrm{c}}\right)\left[\sqrt{\eta_{\mathrm{g}}-\eta_{\mathrm{c}}}-\sqrt{\eta-\eta_{\mathrm{c}}}\right]+} \\
& +\exp \left(-\eta_{\mathrm{g}}\right)\left[2 \sqrt{\frac{\eta_{\mathrm{g}}-\eta}{\pi}}-1\right]-\exp \left(\eta_{\mathrm{g}}\right)\left[1+\operatorname{erf}\left(\sqrt{\eta_{\mathrm{g}}-\eta_{\mathrm{c}}}\right)\right]+2 \exp \left(\eta_{\mathrm{g}}\right) \cdot \operatorname{erf}\left(\sqrt{\eta_{\mathrm{g}}}\right)-4 \sqrt{\frac{\eta_{\mathrm{g}}}{\pi}}
\end{aligned}
$$

Par des raisonnements analogues, on démontre les résultats analytiques suivants :

CAS $a .1$. - Potentiel monotone avec $0<\eta_{\mathrm{c}}<\eta_{\mathrm{g}}$ (Fig. 2a.1)

$$
\begin{gathered}
n_{\mathrm{e}}=0,5 \exp (\eta)[1-\operatorname{erf}(\sqrt{\eta})] \\
n_{\mathrm{i}}=0,5 \exp (-\eta)\left[1-\operatorname{erf}\left(\sqrt{\eta_{\mathrm{g}}-\eta}\right)\right] \\
F(\eta)=\exp (\eta)[1-\operatorname{erf}(\sqrt{\eta})]+2 \sqrt{\eta / \pi}+\exp (-\eta)\left[1-\operatorname{erf}\left(\sqrt{\eta_{\mathrm{g}}-\eta}\right)\right]+2 \exp \left(-\eta_{\mathrm{g}}\right) \cdot \sqrt{\left(\eta_{\mathrm{g}}-\eta\right) / \pi}- \\
-\exp \left(\eta_{\mathrm{g}}\right)\left[1-\operatorname{erf}\left(\sqrt{\eta_{\mathrm{g}}}\right)\right]-2 \sqrt{\eta_{\mathrm{g}} / \pi}-\exp \left(-\eta_{\mathrm{g}}\right)+F\left(\eta_{\mathrm{g}}\right) .
\end{gathered}
$$

CAS $a$.2. - Potentiel monotone avec $\eta_{\mathrm{c}}<0<\eta_{\mathrm{g}}$ (Fig. 2a.2). $n_{\mathrm{i}}$ est donné par la relation (14).

Région 1, $0<\eta<\eta_{\mathrm{g}}$.

$$
\begin{gathered}
n_{\mathrm{e} 1}=0,5 \exp (\eta)\left[1-2 \operatorname{erf}(\sqrt{\eta})+\operatorname{erf}\left(\sqrt{\eta-\eta_{\mathrm{c}}}\right)\right] \\
F_{\mathrm{I}}(\eta)=H(\eta)-2 \exp (\eta) \operatorname{erf}(\sqrt{\eta})+4 \sqrt{\eta / \pi}+F\left(\eta_{\mathrm{g}}\right)
\end{gathered}
$$

Région 2, $\eta_{\mathrm{c}}<\eta<0$.

$$
\begin{gathered}
n_{\mathrm{e} 2}=0,5 \exp (\eta)\left[1+\operatorname{erf}\left(\sqrt{\eta-\eta_{\mathrm{c}}}\right)\right] \\
F_{\mathrm{II}}(\eta)=H(\eta)+F\left(\eta_{\mathrm{g}}\right)
\end{gathered}
$$

où $H(\eta)$ est la fonction donnée précédemment.

CAS $a$.3. - Potentiel monotone avec $\eta_{\mathrm{c}}<\eta_{\mathrm{g}}<0$ (Fig. 2a.3). $n_{\mathrm{e}}$ donné par la relation (16)

$$
\begin{gathered}
n_{\mathrm{i}}=0,5 \exp (-\eta)[1-\operatorname{erf}(\sqrt{-\eta})] \\
F(\eta)=\exp (\eta)\left[1+\operatorname{erf}\left(\sqrt{\eta-\eta_{\mathrm{c}}}\right)\right]-2 \exp \left(\eta_{\mathrm{c}}\right) \sqrt{\left(\eta-\eta_{\mathrm{c}}\right) / \pi} \\
+\exp (-\eta)[1-\operatorname{erf}(\sqrt{-\eta})]+2 \sqrt{-\eta / \pi}-\exp \left(\eta_{\mathrm{g}}\right)\left[1+\operatorname{erf}\left(\sqrt{\eta_{\mathrm{g}}-\eta_{\mathrm{c}}}\right)\right] \\
+2 \exp \left(\eta_{\mathrm{c}}\right) \sqrt{\left(\eta_{\mathrm{g}}-\eta_{\mathrm{c}}\right) / \pi}-2 \sqrt{-\eta_{\mathrm{g}} / \pi}-\exp \left(-\eta_{\mathrm{g}}\right)\left[1-\operatorname{erf}\left(\sqrt{-\eta_{\mathrm{g}}}\right)\right]+F\left(\eta_{\mathrm{g}}\right) .
\end{gathered}
$$


CAS $b .1$. - Potentiel avec maximum $\eta_{\mathrm{m}}$ tel que $\eta_{\mathrm{m}}<\stackrel{\circ}{\mathrm{c}}<\eta_{\mathrm{g}}$ (Fig. 2b.1). $n_{\mathrm{i}}$ est donné par la relation (14).

Région $1,0<\eta<\eta_{\mathrm{g}}$.

$$
\begin{gathered}
n_{\mathrm{e} 1}=0,5 \exp (\eta)\left[1-2 \operatorname{erf}(\sqrt{\eta})+\operatorname{erf}\left(\sqrt{\eta-\eta_{\mathrm{m}}}\right)\right] \\
F_{1}(\eta)=F_{2}(\eta)-2 \exp (\eta) \operatorname{erf}(\sqrt{\eta})+4 \sqrt{\eta / \pi}
\end{gathered}
$$

Région 2, $\eta_{\mathrm{m}}<\eta<0$.

$$
\begin{gathered}
n_{\mathrm{e} 2}=0,5 \exp (\eta)\left[1+\operatorname{erf}\left(\sqrt{\eta-\eta_{\mathrm{m}}}\right)\right] \\
F_{2}(\eta)=\exp (\eta)\left[1+\operatorname{erf}\left(\sqrt{\eta-\eta_{\mathrm{m}}}\right)\right]+\exp (-\eta)\left[1-\operatorname{erf}\left(\sqrt{\eta_{\mathrm{g}}-\eta}\right)\right]+2 \exp \left(-\eta_{\mathrm{g}}\right)\left[\sqrt{\left(\eta_{\mathrm{g}}-\eta\right) / \pi}-\sqrt{\left(\eta_{\mathrm{g}}-\eta_{\mathrm{m}}\right) / \pi}\right]- \\
-\exp \left(\eta_{\mathrm{m}}\right)\left[2 \sqrt{\left(\eta-\eta_{\mathrm{m}}\right) / \pi}+1\right]-\exp \left(-\eta_{\mathrm{m}}\right)\left[1-\operatorname{erf}\left(\sqrt{\eta_{\mathrm{g}}-\eta_{\mathrm{m}}}\right)\right]
\end{gathered}
$$

Région 3, $\eta_{\mathrm{m}}<\eta<\eta_{\mathrm{c}}$.

$$
\begin{gathered}
n_{\mathrm{e} 3}=0,5 \exp (\eta)\left[1-\operatorname{erf}\left(\sqrt{\eta-\eta_{\mathrm{m}}}\right)\right] \\
F_{3}(\eta)=\exp (\eta)\left[1-\operatorname{erf}\left(\sqrt{\eta-\eta_{\mathrm{m}}}\right)\right]+\exp (-\eta)\left[1-\operatorname{erf}\left(\sqrt{\eta_{\mathrm{g}}-\eta}\right)\right]+\exp \left(\eta_{\mathrm{m}}\right)\left[2 \sqrt{\left(\eta-\eta_{\mathrm{m}}\right) / \pi}-1\right] \\
-\exp \left(-\eta_{\mathrm{m}}\right)\left[1-\operatorname{erf}\left(\sqrt{\eta_{\mathrm{g}}-\eta_{\mathrm{m}}}\right)\right]+2 \exp \left(-\eta_{\mathrm{g}}\right)\left(\sqrt{\left(\eta_{\mathrm{g}}-\eta\right) / \pi}-\sqrt{\left(\eta_{\mathrm{g}}-\eta_{\mathrm{m}}\right) / \pi}\right)
\end{gathered}
$$

CAS b.2. - Potentiel avec minimum $\eta_{\mathrm{m}}$ tel que $0<\eta_{\mathrm{m}}<\eta_{\mathrm{c}}<\eta_{\mathrm{g}}$ (Fig. 2b.2). $n_{\mathrm{e}}$ donné par la relation (13) et $n_{\mathrm{i}}$ par la relation (14)

$$
\begin{aligned}
F(\eta)=\exp (\eta)[1-\operatorname{erf} & (\sqrt{\eta})]-\exp \left(\eta_{\mathrm{m}}\right)\left[1-\operatorname{erf}\left(\sqrt{\eta_{\mathrm{m}}}\right)\right]+ \\
& +2\left(\sqrt{\eta / \pi}-\sqrt{\eta_{\mathrm{m}} / \pi}\right)+\exp (-\eta)\left[1-\operatorname{erf}\left(\sqrt{\eta_{\mathrm{g}}-\eta}\right)\right]-\exp \left(-\eta_{\mathrm{m}}\right)\left[1-\operatorname{erf}\left(\sqrt{\eta_{\mathrm{g}}-\eta_{\mathrm{m}}}\right)\right] \\
+ & 2 \exp \left(-\eta_{\mathrm{g}}\right)\left[\sqrt{\left(\eta_{\mathrm{g}}-\eta\right) / \pi}-\sqrt{\left(\eta_{\mathrm{g}}-\eta_{\mathrm{m}}\right) / \pi}\right] .
\end{aligned}
$$

CaS $c .1$. - Potentiel avec maximum $\eta_{\mathrm{p}}$ tel que $\eta_{\mathrm{c}}<\eta_{\mathrm{g}}<\eta_{\mathrm{p}}<0$ (Fig. 2c.1). $n_{\mathrm{i}}$ donné par la relation (17). Région $1, \eta_{\mathrm{g}}<\eta$.

$$
\begin{gathered}
n_{\mathrm{e} 1}=0,5 \exp (\eta)\left[1+\operatorname{erf}\left(\sqrt{\eta-\eta_{\mathrm{c}}}\right)-2 \operatorname{erf}\left(\sqrt{\eta-\eta_{\mathrm{g}}}\right)\right] \\
F_{1}(\eta)=F_{2}(\eta)+4 \exp \left(\eta_{\mathrm{g}}\right) \sqrt{\left(\eta-\eta_{\mathrm{g}}\right) / \pi}-2 \exp (\eta) \operatorname{erf}\left(\sqrt{\eta-\eta_{\mathrm{g}}}\right) .
\end{gathered}
$$

Région 2, $\eta<\eta_{\mathrm{g}} \cdot n_{\mathrm{e} 2}$ donné par la relation (16)

$$
\begin{aligned}
F_{2}(\eta)=\exp (\eta) & {\left[1+\operatorname{erf}\left(\sqrt{\eta-\eta_{\mathrm{c}}}\right)\right]+2 \exp \left(\eta_{\mathrm{c}}\right)\left[\sqrt{\left(\eta_{\mathrm{p}}-\eta_{\mathrm{c}}\right) / \pi}-\sqrt{\left(\eta-\eta_{\mathrm{c}}\right) / \pi}\right]+\exp (-\eta)[1-\operatorname{erf}(\sqrt{-\eta})]+} \\
& +2\left[\sqrt{-\eta / \pi}-\sqrt{-\eta_{\mathrm{p}} / \pi}\right]-4 \exp \left(\eta_{\mathrm{g}}\right) \sqrt{\left(\eta_{\mathrm{p}}-\eta_{\mathrm{g}}\right) / \pi}-\exp \left(\eta_{\mathrm{p}}\right)\left[1+\operatorname{erf}\left(\sqrt{\eta_{\mathrm{p}}-\eta_{\mathrm{c}}}\right)\right]-\exp \left(-\eta_{\mathrm{p}}\right) \\
& \times\left[1-\operatorname{erf}\left(\sqrt{-\eta_{\mathrm{p}}}\right)\right]+2 \exp \left(\eta_{\mathrm{p}}\right) \operatorname{erf}\left(\sqrt{\eta_{\mathrm{p}}-\eta_{\mathrm{g}}}\right)
\end{aligned}
$$

CAS c.2. - Potentiel avec maximum $\eta_{\mathrm{p}}$ tel que $\eta_{\mathrm{c}}<\eta_{\mathrm{g}}<0<\eta_{\mathrm{p}}$ (Fig. 2c.2).

Région $1, \eta_{\mathrm{g}}<\eta<0$. $n_{\mathrm{e} 1}$ donné par la relation (18)

$$
\begin{gathered}
n_{\mathrm{i} 1}=0,5 \exp (-\eta)\left[1+\operatorname{erf}\left(\sqrt{\eta_{\mathrm{p}}-\eta}\right)-2 \operatorname{erf}(\sqrt{-\eta})\right] \\
F_{1}(\eta)=F_{2}(\eta)-2 \exp (-\eta) \operatorname{erf}(\sqrt{-\eta})+4 \sqrt{-\eta / \pi}
\end{gathered}
$$

Région 2, $0<\eta<\eta_{\mathrm{p}}$. $n_{\mathrm{e} 2}$ donné par la relation (18)

$$
\begin{gathered}
n_{\mathrm{i} 2}=0,5 \exp (-\eta)\left[1+\operatorname{erf}\left(\sqrt{\eta_{\mathrm{p}}-\eta}\right)\right] \\
F_{2}(\eta)=\exp (\eta)\left[1+\operatorname{erf}\left(\sqrt{\eta-\eta_{\mathrm{c}}}\right)\right]-\exp \left(\eta_{\mathrm{p}}\right)\left[1+\operatorname{erf}\left(\sqrt{\eta_{\mathrm{p}}-\eta_{\mathrm{c}}}\right)\right]- \\
-2 \exp \left(\eta_{\mathrm{c}}\right)\left[\sqrt{\left(\eta-\eta_{\mathrm{c}}\right) / \pi}-\sqrt{\left(\eta_{\mathrm{p}}-\eta_{\mathrm{c}}\right) / \pi}\right]-2 \exp (\eta) \operatorname{erf}\left(\sqrt{\eta-\eta_{\mathrm{g}}}\right) \\
+2 \exp \left(\eta_{\mathrm{p}}\right) \operatorname{erf}\left(\sqrt{\eta_{\mathrm{p}}-\eta_{\mathrm{g}}}\right)+4 \exp \left(\eta_{\mathrm{g}}\right)\left[\sqrt{\left(\eta-\eta_{\mathrm{g}}\right) / \pi}-\sqrt{\left(\eta_{\mathrm{p}}-\eta_{\mathrm{g}}\right) / \pi}\right] \\
+\exp (-\eta)\left[1+\operatorname{erf}\left(\sqrt{\eta_{\mathrm{p}}-\eta}\right)\right]-\exp \left(-\eta_{\mathrm{p}}\right)\left[1+2 \sqrt{\left(\eta_{\mathrm{p}}-\eta\right) / \pi}\right] .
\end{gathered}
$$


Région 3, $\eta_{\mathrm{g}}<\eta<\eta_{\mathrm{p}} . n_{\mathrm{e} 3}$ donné par la relation (18)

$$
\begin{gathered}
n_{\mathrm{i} 3}=0,5 \exp (-\eta)\left[1-\operatorname{erf}\left(\sqrt{\eta_{\mathrm{p}}-\eta}\right)\right] \\
F_{3}(\eta)=F_{2}(\eta)-2 \exp (-\eta) \operatorname{erf}\left(\sqrt{\eta_{\mathrm{p}}-\eta}\right)+4 \exp \left(-\eta_{\mathrm{p}}\right) \sqrt{\left(\eta_{\mathrm{p}}-\eta\right) / \pi} .
\end{gathered}
$$

Région 4, $\eta_{\mathrm{c}}<\eta<\eta_{\mathrm{g}}, n_{\mathrm{e} 4}$ donné par la relation (16)

$$
\begin{aligned}
F_{4}(\eta)=F_{2}(\eta)+2 \exp (\eta) \operatorname{erf}\left(\sqrt{\eta-\eta_{\mathrm{g}}}\right)-4 \exp \left(\eta_{\mathrm{g}}\right) \sqrt{\left(\eta-\eta_{\mathrm{g}}\right) / \pi}-2 \exp (-\eta) \operatorname{erf}\left(\sqrt{\eta_{\mathrm{p}}-\eta}\right)+ & +4 \exp \left(-\eta_{\mathrm{p}}\right) \sqrt{\left(\eta_{\mathrm{p}}-\eta\right) / \pi} . \\
& +4 .
\end{aligned}
$$
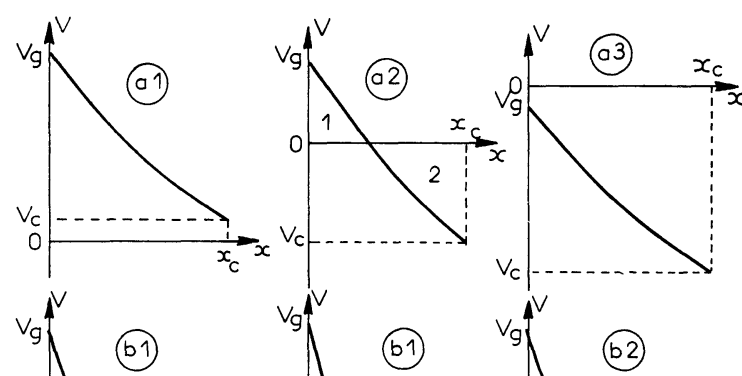

$v_{c} v_{c}$
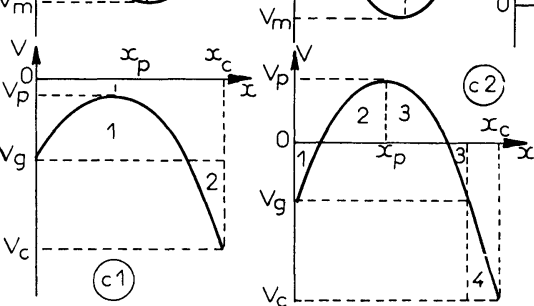

(b2)

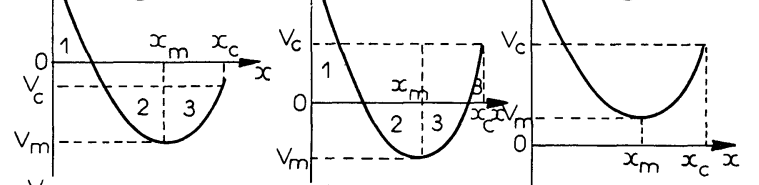

FIG. 2. - Les différents cas de potentiel envisagés.

Remarque. - Nous n'avons traité que le cas où $\eta_{\mathrm{c}}<\eta_{\mathrm{g}}$, le cas inverse se déduit de celui-ci par échange des conclusions entre ions et électrons. Par ailleurs, il faut noter qu'il existe d'autres cas de distribution de potentiel que ceux étudiés ici, lesquels ont été déterminés numériquement en fonction des valeurs expérimentales de $V_{\mathrm{c}}$ et $V_{\mathrm{g}}$ [5]. Il faut néanmoins remarquer que certaines répartitions sont impossibles; ainsi, nous avons constaté numériquement qu'une répartition comportant un minimum, lorsque $V_{\mathrm{c}}$ et $V_{\mathrm{g}}$ sont tous deux négatifs, ne pouvait être réalisée avec les valeurs habituellement prises pour les autres paramètres (densités et températures).

4. Etude des courants collectés. - La densité de courant $J$ dans le plan situé à la distance $x$ de la grille est donnée par la relation :

$$
J(x)=J_{\mathrm{e}}(x)+J_{\mathrm{i}}(x)
$$

où les densités de courant électronique et ionique se calculent à partir des fonctions de distribution :

$$
J_{\mathrm{j}}(x)=\varepsilon_{\mathrm{j}} e n_{0} \int_{\text {vitesse des particules } j} v f_{\mathrm{j}}(x, v) \mathrm{d} v
$$

où $j=e$ ou $i$.
L'intégration se faisant sur toutes les vitesses possibles pour chaque type de particules, lesquelles dépendent de la répartition de potentiel, les densités de courant en seront aussi fonction.

Ainsi, à partir des valeurs précédentes, et en posant $J_{\mathrm{js}}=\varepsilon_{\mathrm{j}} n_{0} e\left(k T / 2 \pi m_{\mathrm{j}}\right)^{1 / 2}$ (avec $j=e$ ou $i$ ) comme étant les densités électronique et ionique dans le plasma situé devant la grille, nous obtenons, pour chacun des cas envisagés précédemment, les résultats suivants :

Cas a.1.

$$
J_{\mathrm{e}}(x)=J_{\mathrm{es}} \text { et } J_{\mathrm{i}}(x)=J_{\mathrm{is}} \exp \left(-\eta_{\mathrm{g}}\right) .
$$

Cas a.2.

$$
J_{\mathrm{e}}(x)=J_{\mathrm{es}} \exp \left(\eta_{\mathrm{c}}\right) \text { et } J_{\mathrm{i}}(x)=J_{\mathrm{is}} \exp \left(-\eta_{\mathrm{g}}\right) .
$$

Cas a.3.

$$
J_{\mathrm{e}}(x)=J_{\mathrm{es}} \exp \left(\eta_{\mathrm{c}}\right) \quad \text { et } \quad J_{\mathrm{i}}(x)=J_{\text {is }} .
$$

Cas b.1.

$$
J_{\mathrm{e}}(x)=J_{\mathrm{es}} \exp \left(\eta_{\mathrm{m}}\right) \text { et } J_{\mathrm{i}}(x)=J_{\mathrm{is}} \exp \left(-\eta_{\mathrm{g}}\right) \text {. }
$$

Cas b.2.

$$
J_{\mathrm{e}}(x)=J_{\mathrm{es}} \text { et } J_{\mathrm{i}}(x)=J_{\text {is }} \exp \left(-\eta_{\mathrm{g}}\right) .
$$

Cas c.1.

$$
J_{\mathrm{e}}(x)=J_{\mathrm{es}} \exp \left(\eta_{\mathrm{c}}\right) \quad \text { et } \quad J_{\mathrm{i}}(x)=J_{\text {is }} .
$$

Cas c.2.

$$
J_{e}(x)=J_{\text {es }} \exp \left(\eta_{\mathrm{c}}\right) \text { et } \quad J_{\mathrm{i}}(x)=J_{\mathrm{is}} \exp \left(-\eta_{\mathrm{p}}\right) .
$$

Il est intéressant de constater que les expressions de $J_{\mathrm{e}}$ ou $J_{\mathrm{i}}$ ne dépendent pas de $x$, et qu'ainsi la conservation des densités de courant est bien vérifiée. Par contre, les valeurs diffèrent suivant le type de répartition de potentiel envisagée et la sonde collectera un courant, qui selon le cas, dépendra de $V_{c}$, de $V_{\mathrm{g}}$, de $V_{\mathrm{m}}$ ou de $V_{\mathrm{p}}$.

5. Résultats numériques. - Nous avons donc poursuivi notre étude par la résolution numérique dans les conditions physiques suivantes : la distance interélectrode étant de $1,1 \mathrm{~mm}$, l'énergie $k T$ des ions et des électrons étant prise étage à $0,2 \mathrm{eV}$ et la densité $N_{0}$ à $2 \times 10^{9} \mathrm{~cm}^{-3}$, la distance réduite est alors égale à 15 , la longueur de Debye valant $0,0733 \mathrm{~mm}$. 
Pour mener à bien ces développements numériques, il s'est avéré que dans certains calculs d'intégrales une coupure était nécessaire. En effet, lorsque l'une des bornes annule le dénominateur en $\sqrt{F(\eta)}$, le calcul numérique direct de l'intégrale correspondante est impossible bien qu'elle soit analytiquement convergente. Nous avons alors introduit un paramètre $\lambda$ qui était ajouté ou retranché à cette borne $\eta$, suivant les cas.

Ainsi, par exemple dans le cas $b .1$, la condition à remplir (9) s'écrit :

$$
\xi_{\mathrm{c}}=-\int_{\eta_{\mathrm{g}}}^{0} \frac{\mathrm{d} \eta}{\sqrt{F_{1}(\eta)}}-\int_{0}^{\eta_{\mathrm{m}}} \frac{\mathrm{d} \eta}{\sqrt{F_{2}(\eta)}}+\int_{\eta_{\mathrm{m}}}^{\eta_{\mathrm{c}}} \frac{\mathrm{d} \eta_{\mathrm{d}}}{\sqrt{F_{3}(\eta)}} .
$$

Or, $F_{2}\left(\eta_{\mathrm{m}}\right)=F_{3}\left(\eta_{\mathrm{m}}\right)=0$.

Nous avons alors modifié cette condition de la façon suivante :

$$
\begin{aligned}
\xi_{\mathrm{c}}=-\int_{\eta_{\mathrm{g}}}^{0} \frac{\mathrm{d} \eta}{\sqrt{F_{1}(\eta)}} & -\int_{0}^{\eta_{\mathrm{m}}+\lambda} \frac{\mathrm{d} \eta}{\sqrt{F_{2}(\eta)}}+ \\
& +\int_{\eta_{\mathrm{m}}+\lambda}^{\eta_{\mathrm{c}}} \frac{\mathrm{d} \eta}{\sqrt{F_{3}(\eta)}}+R\left(\eta_{\mathrm{m}}, \eta_{\mathrm{g}}, \lambda\right)
\end{aligned}
$$

où le reste $R$ développé au premier ordre en $\lambda$ autour de $\eta_{\mathrm{m}}$ prend la forme :

$$
\begin{aligned}
& R\left(\eta_{\mathrm{m}}, \eta_{\mathrm{g}}, \lambda\right)= \\
= & 4 \sqrt{\lambda}\left[\exp \left(\eta_{\mathrm{m}}\right)-\exp \left(-\eta_{\mathrm{m}}\right)\left\{1-\operatorname{erf}\left(\sqrt{\eta_{\mathrm{g}}-\eta_{\mathrm{m}}}\right)\right\}\right]^{-\frac{1}{2}} .
\end{aligned}
$$

Dans tous les cas, où un tel problème de borne annulant le dénominateur s'est posé, nous avons tenu compte de la valeur de ce genre de reste dans les résultats que nous exposons.

Pour le calcul des fonctions spéciales intervenant dans les expressions précédentes, nous avons utilisé les développements donnés par les références [6], [7] et [8].

Dans un premier temps, nous avons pris une différence $\eta_{\mathrm{g}}-\eta_{\mathrm{c}}$ constante et égale à 6 . L'expérience numérique montre que les résultats ne convergent vers la valeur désirée que si $\lambda$ est suffisamment petit, nous avons ainsi donné à $\lambda$ la valeur de $10^{-6}$ pour l'ensemble de nos calculs.

La figure 3 représente les variations du potentiel $\eta-\eta_{\mathrm{g}}$ en fonction de la distance réduite $\xi$ pour quelques valeurs de $\eta_{\mathrm{g}}$, nous avons déterminé celles qui annulaient le champ électrique sur la grille et sur le collecteur, et nous avons trouvé respectivement $-1,868$ et $+2,692$.

$$
\begin{array}{r}
\mathrm{Si}-1,868<\eta_{\mathrm{g}}<+2,692 \text { le potentielest monotone, } \\
\eta_{\mathrm{g}}<-1,868 \text { le potentiel présente un } \\
\quad \text { maximum, } \\
\eta_{\mathrm{g}}>+2,692 \text { le potentiel présente un } \\
\text { minimum. }
\end{array}
$$

Lorsque le potentiel est monotone, nous avons au voisinage de la grille une charge d'espace électronique qui diminue lorsque $\eta_{\mathrm{g}}$ décroît de 2,692 à $-1,868$

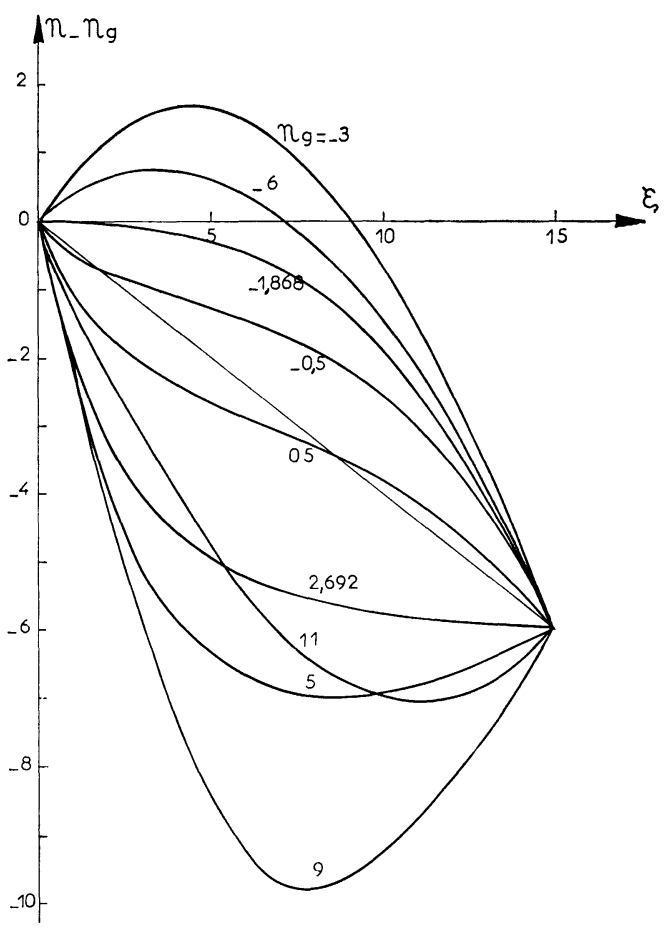

Fig. 3. - Répartition de potentiel pour différentes valeurs de $\eta_{\mathrm{g}}$ avec $\eta_{\mathrm{g}}-\eta_{\mathrm{c}}=6$ et $\xi_{\mathrm{c}}=15$.

au profit d'une charge d'espace ionique qui augmente près du collecteur.

Si $\eta_{\mathrm{g}}>2,868$ et croissant, nous constatons que $\eta_{\mathrm{c}}-\eta_{\mathrm{m}}$ croît puis décroît ensuite. Nous pouvons interpréter ce résultat de la façon suivante:

Les électrons franchissent la grille avec une énergie cinétique d'autant plus grande que $\eta_{\mathrm{g}}$ est positif. Mais tant que leur vitesse n'est pas suffisamment importante, ils ont tendance à s'accumuler dans l'espace interélectrode, même si $\eta_{\mathrm{c}}$ est positif, et $\eta_{\mathrm{c}}-\eta_{\mathrm{m}}$ augmente. Dès que leur vitesse devient élevée, c'està-dire pour $\eta_{\mathrm{g}}$ grand, le potentiel retardateur grillecollecteur a moins d'influence et $\eta_{\mathrm{c}}-\eta_{\mathrm{m}}$ diminue.

De même, si $\eta_{\mathrm{g}}<-1,868$ et décroissant, $\eta_{\mathrm{p}}-\eta_{\mathrm{g}}$ croît d'abord puis décroît; en effet, les ions sont accélérés après avoir franchi la grille et leur vitesse après celle-ci est d'autant plus importante que $\eta_{\mathrm{g}}$ est plus négatif. Mais ils peuvent néanmoins s'accumuler eux aussi au voisinage de la grille si $\eta_{\mathbf{g}}$ n'est pas assez négatif et $\eta_{\mathrm{p}}-\eta_{\mathrm{g}}$ augmente. Lorsque $\eta_{\mathrm{c}}$ est très négatif les ions sont collectés encore plus facilement et $\eta_{\mathrm{p}}-\eta_{\mathrm{g}}$ diminue.

Notons que $\eta_{\mathrm{c}}-\eta_{\mathrm{m}}$ atteint de plus grandes valeurs que $\eta_{\mathrm{p}}-\eta_{\mathrm{g}}$. En effet, les électrons sont ralentis dans l'espace interélectrode alors que les ions sont accélérés.

Sur la figure $4 a$ nous avons représenté les variations du champ électrique réduit en fonction de la distance réduite pour différentes valeurs de $\eta_{\mathrm{g}}$ positif pour une différence $\eta_{\mathrm{g}}-\eta_{\mathrm{c}}$ constante et égale à 6 .

Lorsque $\eta_{\mathrm{g}}$ croît de 0 à 2,692, valeur pour laquelle le champ électrique est nul sur le collecteur, $\mathrm{d} \eta / \mathrm{d} \xi$ 


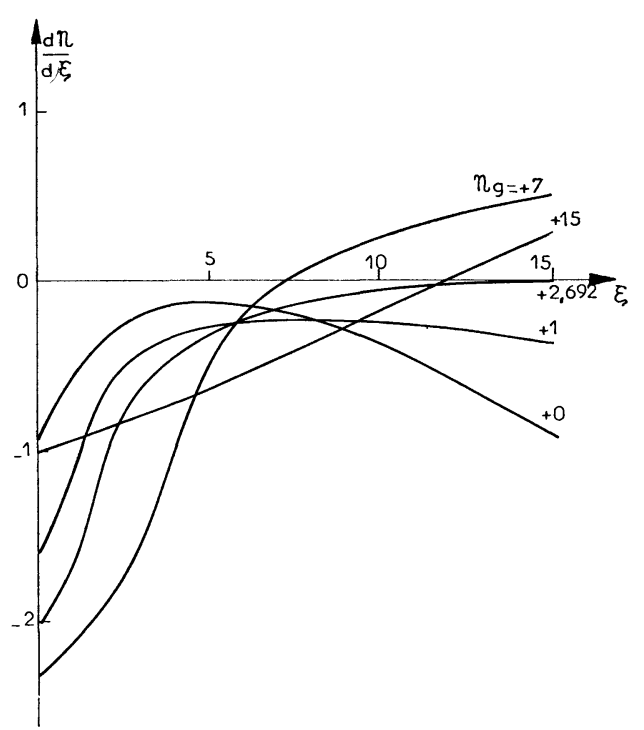

(a)

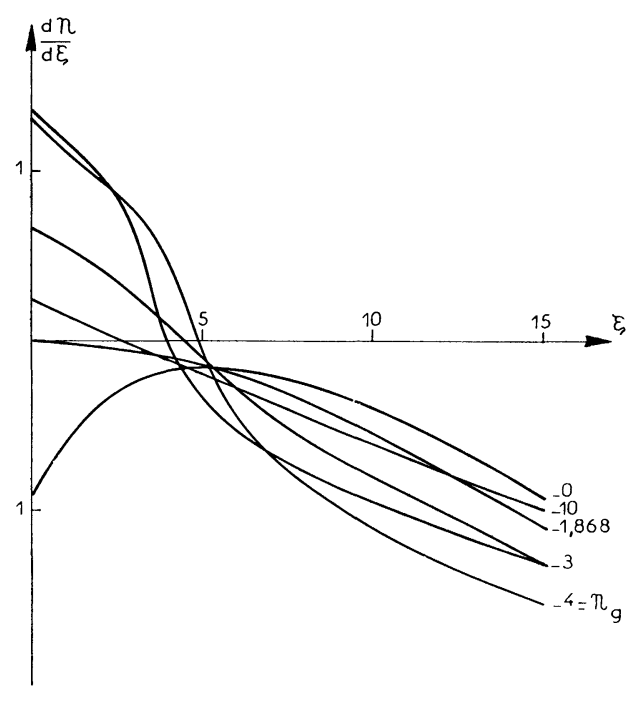

(b)

Fig. 4. - Champ électrique réduit en fonction de $\xi$ avec $\eta_{\mathrm{g}}-\eta_{\mathrm{c}}=6$ et $\left.\xi_{\mathrm{c}}=15: a\right)$ pour différentes valeurs positives de $\left.\eta_{\mathrm{g}} ; b\right)$ pour différentes valeurs négatives de $\eta_{\mathrm{g}}$.

présente un maximum dans l'espace interélectrode, nous sommes dans le domaine où la répartition de potentiel est monotone. Puis lorsque $\eta_{\mathrm{g}}$ croît de 2,69 à 15 et au-delà, le champ électrique demeure croissant avec $\xi$ et change par suite de la présence du minimum de potentiel.

Sur la figure $4 b$ nous avons tracé $\mathrm{d} \eta / \mathrm{d} \xi$ en fonction de $\xi$ pour des valeurs négatives de $\eta_{\mathrm{g}}$. Lorsque $\eta_{\mathrm{g}}$ décroît de 0 à $-1,868$, le champ électrique présente un maximum et la répartition de potentiel est monotone. Quand $\eta_{\mathrm{g}}<-1,868$, le champ électrique change de signe et demeure décroissant dans l'espace grillecollecteur.

Les figures 5 et 6 représentent l'évolution des densités électronique et ionique réduites en fonction de la distance réduite $\xi$. La forme des courbes dépend

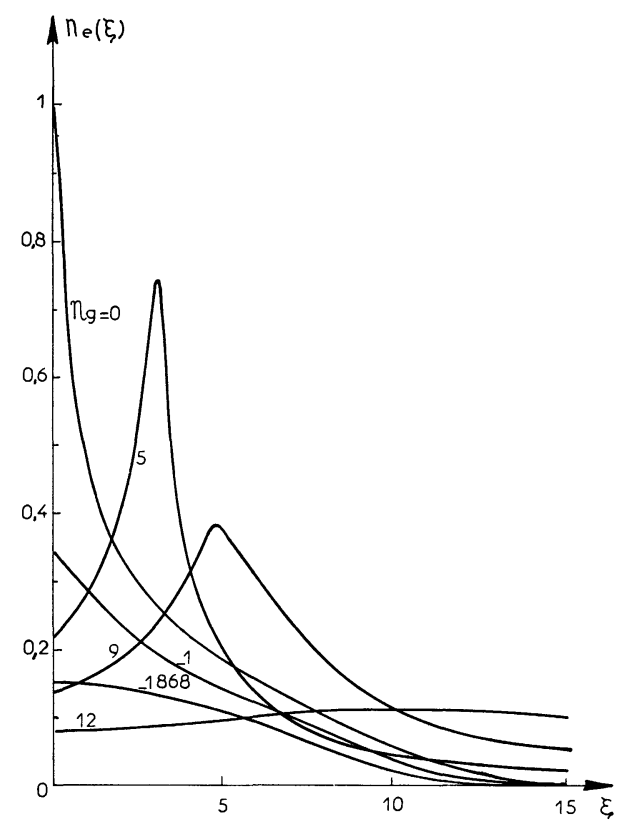

Fig. 5. - Densité électronique réduite en fonction de $\xi$ avec $\eta_{\mathrm{g}}-\eta_{\mathrm{c}}=6$ et $\xi_{\mathrm{c}}=15$ pour différentes valeurs de $\eta_{\mathrm{g}}$.

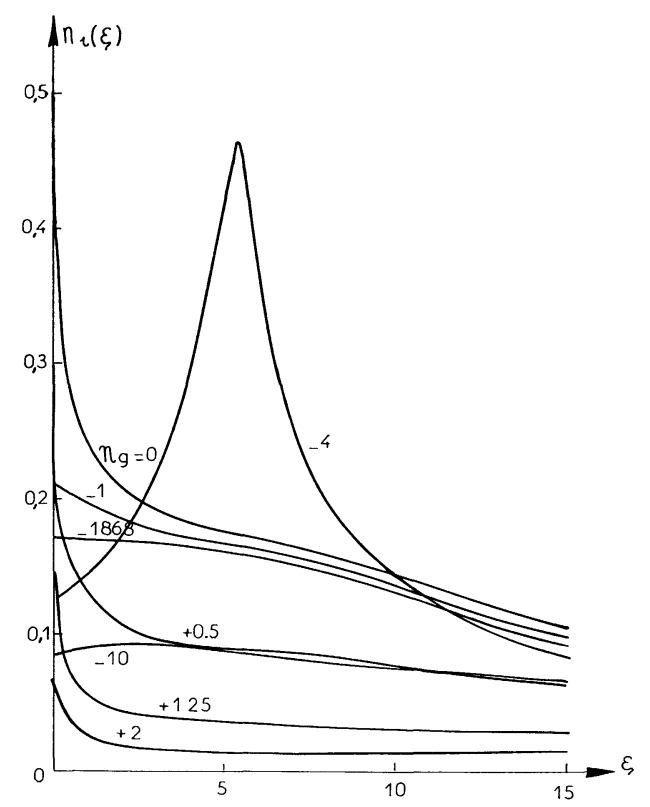

FIG. 6. - Densité ionique dans les mêmes conditions que la figure 5 .

essentiellement du potentiel de grille. La densité électronique diminue rapidement lorsque $\eta_{\mathrm{c}}$ devient très négatif et tend à devenir uniforme dans l'espace interélectrode lorsque $\eta_{\mathrm{g}}$ est positif.

La densité ionique diminue lorsque $\eta_{\mathrm{g}}$ devient positif et tend à rester constante quand $\eta_{\mathrm{g}}$ est très négatif. Les courbes mettent clairement en évidence les différentes possibilités d'existence de charges d'espace.

Le problème que nous nous sommes posés alors est le suivant : étant donné des conditions expérimentales définies par $\eta_{\mathrm{g}}, \eta_{\mathrm{c}}$ et $\xi_{\mathrm{c}}$, la répartition de potentiel 
dans l'espace interélectrode est-elle monotone, avec maximum ou avec minimum ? Pour répondre directement à cette question, nous avons cherché à diviser le plan $\left(\eta_{\mathrm{g}}, \eta_{\mathrm{g}}-\eta_{\mathrm{c}}\right)$ en trois régions, chacune de ces régions correspondant à un des trois types de répartition possible.

Les autres conditions étant les mêmes que précédemment pour différentes valeurs de la distance réduite $\xi_{c}$, nous avons alors tracé sur la figure 7 les courbes de changement de type de répartition. Ainsi, à $\eta_{\mathrm{c}}$ et $\eta_{\mathrm{g}}-\eta_{\mathrm{c}}$ fixés, lorsque l'on se déplace sur une parallèle à l'axe des $\eta_{\mathrm{g}}$, c'est-à-dire lorsque $\eta_{\mathrm{g}}$ varie de -5 à +12 , on rencontre les 3 régions correspondant aux 3 types de répartition : en dessous du point $\mathrm{A}, \eta(\xi)$ admet un maximum, entre $\mathrm{A}$ et $\mathrm{B}$, le potentiel est monotone et au-dessus du point $\mathrm{B}$, il admet un minimum.

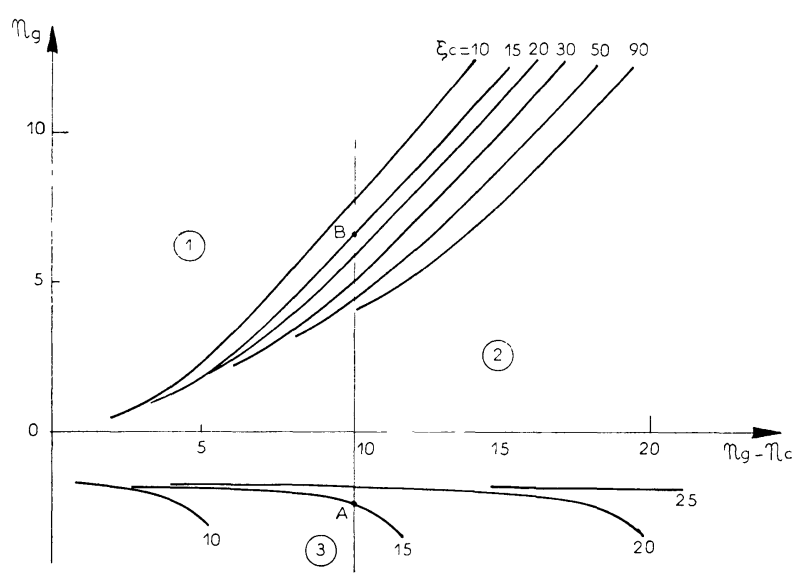

FIG. 7. - Forme du potentiel dans l'espace interélectrode en fonction des paramètres $\eta_{\mathrm{g}}, \eta_{\mathrm{g}}-\eta_{\mathrm{c}}$ et $\xi_{\mathrm{c}}$.

Région 1 : Distribution de potentiel avec minimum.

Région 2: Distribution de potentiel monotone.

Région 3 : Distribution de potentiel avec maximum.

Il apparaît donc que la plage de monotonie du potentiel est d'autant plus importante que $\eta_{\mathrm{g}}-\eta_{\mathrm{c}}$ est plus grand ou que la distance réduite $\xi_{\mathrm{c}}$ est plus petite ; en revanche, les grandes distances interélectrodes ou les petites différences de potentiel $\eta_{\mathrm{g}}-\eta_{\mathrm{c}}$ facilitent l'obtention d'extrema dans la répartition du potentiel. Par ailleurs, la dissymétrie des courbes frontières formées par les points $\mathrm{A}$ et $\mathrm{B}$ provient de ce que la valeur positive de $\eta_{\mathrm{g}}-\eta_{\mathrm{c}}$ conduit à des conclusions différentes mais non opposées suivant que $\eta_{\mathrm{g}}$ est positif ou négatif.

Enfin, il ne nous a pas été possible de prolonger les courbes frontières vers les très petites ou les très grandes valeurs de $\eta_{\mathrm{g}}-\eta_{\mathrm{c}}$; les modèles de répartition de potentiel choisis ne sont plus valables dans ces cas extrêmes, ou bien le sont encore mais alors les méthodes numériques employées sont inadaptées.

6. Discussion et conclusion. - Il est intéressant de confronter nos résultats analytico-numériques à ceux obtenus expérimentalement lors d'une étude antérieure des paramètres ioniques d'un plasma de césium par sonde à grille [9]. Cette étude menée dans un plasma obtenu par décharge et ionisation de contact avait mis en évidence le rôle séparateur de la sonde à grille ; ce rôle ainsi que les conditions dans lesquelles on peut l'obtenir, apparaissent clairement à la lumière de l'étude théorique développée dans ce papier. En effet, les courants collectés par la sonde sont directement proportionnels aux densités de courant calculées au paragraphe 4 ; si l'on envisage le cas monotone (cas $a .1, a .2$ et $a .3$ ) lors de la collection d'une sonde à grille dont on fait varier la tension $V_{\mathrm{c}}$ à $V_{\mathrm{g}}-V_{\mathrm{c}}$ positif constant, on obtient une caractéristique dont la forme est celle donnée sur la figure $8 a$ : la région $\alpha\left(\eta_{\mathrm{c}}\right.$ donc $V_{\mathrm{c}}$ positif) correspond à l'obtention du courant électronique de saturation $J_{\text {es }}$ et d'un

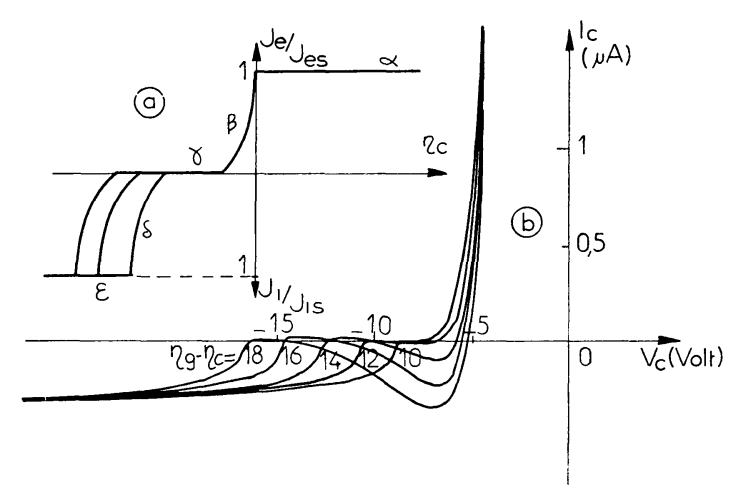

FIG. 8. - Caractéristique de sonde à grille : $a$ ) théorique pour différentes valeurs positives de $\left.\eta_{\mathrm{g}}-\eta_{\mathrm{c}} ; b\right)$ expérimentale : partie ionique pour $V_{\mathrm{g}}-V_{\mathrm{c}}=10,12, \ldots 18 \mathrm{~V}$.

courant ionique extrêmement faible $J_{\text {is }} \exp \left(-\eta_{\mathrm{g}}\right)$, c'est le cas $a .1$; la région $\beta$ ( $\eta_{\mathrm{c}}$ négatif mais petit) conduit à un courant électronique décroissant puisqu'en $J_{\text {es }} \exp \left(\eta_{\mathrm{c}}\right)$ et un courant ionique toujours très faible, c'est le cas $a .2$; dans la région $\gamma\left(\eta_{\mathrm{c}}\right.$ négatif et $\eta_{\mathrm{g}}$ encore positif avec $\left|\eta_{\mathrm{c}}\right|$ et $\left|\eta_{\mathrm{g}}\right|$ du même ordre de grandeur) les courants collectés sont très petits à cause des facteurs $\exp \left(\eta_{\mathrm{g}}\right)$ et $\exp \left(\eta_{\mathrm{c}}\right)$, cela correspond encore au cas $a .2$; ensuite si $\eta_{\mathrm{g}}$ diminue, le nombre d'ions collectés augmente, le courant électronique restant négligeable, c'est la région $\delta$ qui se termine pour $\eta_{\mathrm{g}}=0$; enfin la région $\varepsilon$ correspond à la collection d'un courant ionique $J_{\text {is }}$ de saturation, indépendant de $\eta_{g}$, le courant électronique restant toujours négligeable, c'est le cas $a$.3. Ainsi la séparation des charges est réalisée lorsqu'existe la région $\gamma$ dont l'étendue dépend directement de la différence $\eta_{\mathrm{g}}-\eta_{\mathrm{c}}$; tout ceci ajouté au phénomène d'apparition d'extrema, favorisé par les petites valeurs de $\eta_{\mathrm{g}}-\eta_{\mathrm{c}}$ (Fig. 7), montre que le bon fonctionnement d'une sonde à grille en séparateur de charges nécessite des valeurs de $\eta_{\mathrm{g}}-\eta_{\mathrm{c}}$ supérieures à 16 , si l'on veut des affaiblissements de courant de l'ordre de 100 dans 
la région $\gamma$; la différence de tension $V_{\mathrm{g}}-V_{\mathrm{c}}$ à appliquer doit donc être supérieur à 16 fois l'énergie thermique des particules. Ceci explique et confirme les résultats expérimentaux [9] obtenus en plasma de césium ; ainsi pour un plasma généré dans les conditions suivantes: température d'émetteur $1100 \mathrm{~K}$, pression de césium $10^{-3}$ torr, courant de décharge $100 \mathrm{~mA}$, tension de décharge $3,4 \mathrm{~V}$ et température électronique correspondante $0,6 \mathrm{eV}$, les caractéristiques de sonde à grille possèdent une partie ionique (Fig. $8 b$ ) conforme à ce que prévoit notre théorie: l'accord est bon pour $V_{\mathrm{g}}-V_{\mathrm{c}}=10$ et $12 \mathrm{~V}$, pour les autres valeurs il apparaît un courant parasite d'autant plus intense que $V_{\mathrm{g}}-V_{\mathrm{c}}$ est plus grand; ce courant peut s'interpréter, comme l'a signalé Boyd [10], par une ionisation dans l'espace interélectrode. Pour des valeurs de $V_{\mathrm{g}}-V_{\mathrm{c}}$ plus petites que $10 \mathrm{~V}$, la collection des électrons vient se confondre avec celle des ions, il n'y a plus séparation des charges.

Néanmoins, il faut bien reconnaître que cette théorie est loin de représenter fidèlement le fonction- nement réel d'une sonde à grille. En particulier, trois corrections majeures sont à envisager. La première, qui est de loin la plus importante, concerne les dimensions finies et tridimensionnelles de la sonde; une étude en tenant compte est actuellement en cours et devrait permettre de se rapprocher des conditions expérimentales. La seconde correction est en rapport avec les collisions que nous avons supposées inexistantes, mais qui entrent, dans certains cas, pour une part non négligeable dans la fonction de distribution ; celle-ci, tronquée pour certaines valeurs du potentiel de grille, tendrait, sous l'influence des collisions dans l'espace interélectrodes, à redevenir maxwellienne. Ces deux premières corrections imposent l'utilisation de méthodes de calcul fondamentalement différentes de celle exposée ici. La gaine qui existe devant la grille de la sonde plongée dans le plasma constitue la troisième correction à apporter à cette théorie, malheureusement ce terme de gaine masque encore beaucoup d'ignorance ; l'influence de celle-ci se manifesterait principalement sur les densités ionique et électronique présentes au niveau de la grille.

\section{Bibliographie}

[1] Auer, P. L., J. Appl. Phys. 31 (1960) 2096.

[2] MCIntyre, R. G., J. Appl. Phys. 33 (1962) 2485.

[3] MCIntyre, R. G., Advanced Energy conversion 2 (1962) 405.

[4] Legras, J., Méthodes et Techniques de l'analyse numérique (Dunod, Paris), 1971.

[5] SchirmanN, D., Thèse de $3^{\text {e }}$ Cycle, Nancy.
[6] Abramowitz, M. and Stegun, I., Mathematical functions (Dover Publications Inc., New York).

[7] Cody, W. J., Mathematics of computation 22 (1969) 631.

[8] GaUtschi, W., SIAM, J. Numer. Anal. 7 (1970) 187.

[9] Haug, R., Felden, M. and Schirmann, D., J. Appl. Phys. 39 (1968) 4501.

[10] Boyd, R. L. F., Proc. R. Soc. 201 (1950) 336. 